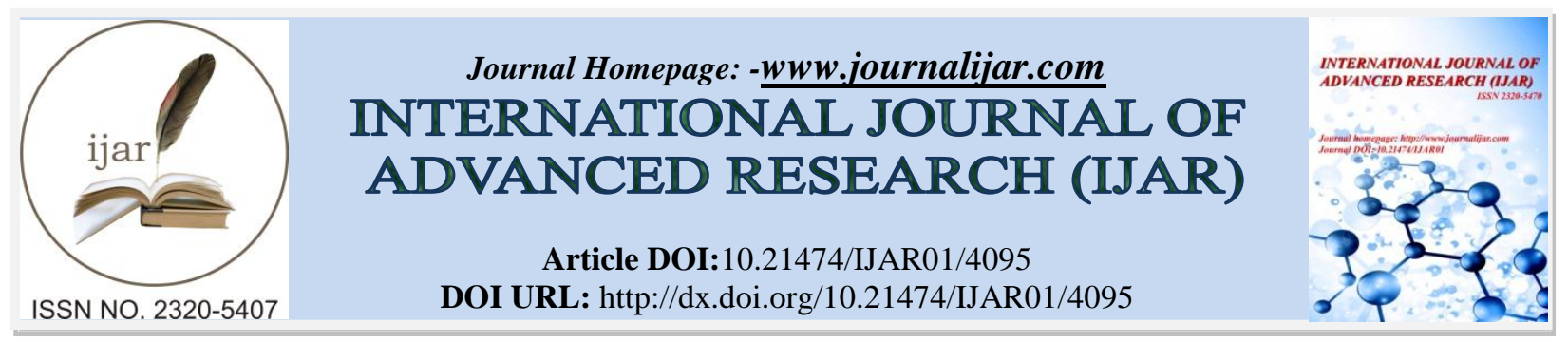

RESEARCH ARTICLE

\title{
IMMUNOLOGICAL IMPACT OF USING INSULIN PUMP WITH TYP1 DIABETES.
}

Mohamd A Alblihed. PhD. MHPE. MSc.

Head of medial biochemistry department, College of medicine, Taif university, Saudi Arabia.

\section{Manuscript Info}

Manuscript History

Received: 04 March 2017

Final Accepted: 08 April 2017

Published: May 2017

\begin{abstract}
This paper is about the full sized keyboard that is implemented using the image processing. In this type of keyboard the image of the keyboard is being projected on any plane surface and a pre calibrated camera tracks the movement of the fingers on the image and detects key being pressed. This keyboard uses embedded system and image processing to let the users work on any surface they want with a higher portability option. This technology has many application in various high tech and industrial sectors where the keyboards would be in sterile and low noise environments for example operation theatres.
\end{abstract}

Copy Right, IJAR, 2016,. All rights reserved.

\section{Introduction}

Type I diabetes is among the chronic diseases affecting a wide range of people in the world today. The condition is characterized by lack of enough insulin in the body to help in the conversation of sugar into energy. People with type I diabetes suffer complications associated with high levels of glucose in the body such as a reduced cytokine response (35). The autoimmune condition causes the immune system to destroy the cells responsible for the production of insulin in the pancreas. The reason behind this reaction by the immune system is unknown thus making it hard for the condition to be preventable. Further, no known cure exists for type I diabetes but several therapies have been developed to ensure that people live near normal lives even with the condition. Type I diabetes represents about $10 \%$ of all diabetes cases in the world and it is prevalent among the young generation (37). The disease can affect people at a very early age and it is characterized by excessive urination, weight loss, blurred vision, and fatigue among other things. People use different mechanisms to keep the supply of the insulin in the body constant. The use of injections and insulin pumps are the most used mechanism in the world today to manage diabetes type I (8).

Lack of insulin in the body can cause many damages and lead to a defect in the immunity system. Without insulin, the body lacks the capacity of converting glucose into energy. As an alternative, the body burns its fats to provide energy thus leading to accumulation of chemicals that are dangerous that life threatening (10). Although eating healthy is ideal in the management of the type I diabetes, once the symptoms are visible, it is impossible to cure the disease. The best one can do is to find a viable therapy that can help in the management of insulin and ensure a close to normal living.

Ketoacidosis is one of the complications that arise when type I diabetes patients do not receive insulin in their systems (29). The condition is caused by excess glucose in the body and the accumulation of chemical substances arising from the burning of fat. Symptoms of ketoacidosis include rapid breathing, abdominal pain, dehydration, and vomiting among other things (26). The condition is life threatening and it should be handled as soon as the symptoms arise. 
Research indicates that type I diabetic people have a higher level of infection in comparison to normal persons. The decreased cytokine response and improved cellular functions affect the immunity levels of the body thus giving room for infections (11). In addition, some organisms thrive well in a high glucose environment; therefore, lack of insulin creates an ideal environment for harmful bacteria to propagate in the body. High blood glucose can lead to the malfunction of the immune system and effect the rate at which the body heals from wounds and other minor problems (33). Further, open wounds are entry points for bacterial, which makes the lack of insulin in the body a double tragedy.

\section{The Immune System}

The function of the immune system is of paramount importance to the body. The immune system keeps the body from disease and it equips the white blood cells to fight against any harmful pathogens in the body (1). Problems with the immune system must be handled with immediate effect to keep the body safe and to allow one to operate normally. The main role of the immune system is to protect the body from tumors, virus, and tumors (9). The action paths used by the system to deal with these threats are multiple and they depend on the problem being handled. The bone marrow, spleen, lymph nodes, and thymus make up the immune system of the body. In a type I diabetes case, the immune system acts against its own mechanism by killing he cells responsible for the production of the insulin (17). The reaction is believed to be caused by a combination of genetic and environmental factors that trigger the abnormal action of the immune system. The T lymphocyte cells start to target and kill cells in the pancreas that produce insulin when the disease is triggered (6). The introduction of insulin in the blood through various mechanisms help to counteract these actions by providing the body with the insulin that cannot be produced naturally in the system thus allowing for proper digestion of food substances (22).

\section{Treatment and Drugs For Type I Diabetes}

Several treatments exist for type I diabetes but all aim at ensuring that there is a constant supply of insulin in the body. The most notable therapies used for the condition include taking insulin, carbohydrate counting, checking blood sugar regularly, healthy eating, and management of weight (32). The constant monitoring of the blood sugar level and the provision of the insulin helps one to keep the body systems as close to normal as possible and prevent complications that arise from the accumulation of chemicals in the body. The treatments ensure that during the daytime, the glucose level is kept under $130 \mathrm{mg} / \mathrm{dl}$ before meals and $180 \mathrm{mg} / \mathrm{dl}$ after meals (38). The management of these therapies is not easy and can seem overwhelming during the first few days. However, research indicates that people have lived near normal lives with type I diabetes regardless of the challenges (7). The different treatments use varied types of insulin including rapid acting, long lasting and intermediate insulin.

Insulin administration. The stomach enzymes interfere with the action of externally administered insulin; therefore, the intervention cannot be administered orally. Most people use injections and insulin pumps to administer insulin into their systems (12). The injection is administered through a needle and syringe or advanced equipment called the insulin pen. The pens are available in many forms to serve a wide range of customers and so are the needles (33). People with injection phobia can use the pen because it looks more comfortable. With the injection, one has to make use of both long acting and rapid insulin to manage the day time and night insulin requirements. Research shows that three injections a day provide the best results so far for people with type I diabetes (37).

The use of insulin pumps has been highly advocated in the world today. The insulin pump is a small device that takes different shapes to suit the needs of varied clients. The device is worn outside the body with a connection to a reservoir that contains the insulin (39). A catheter is inserted under the skin of the patient and the pump is programmed to release a certain amount of insulin into the system. The insulin pump mimics the pancreas. The insulin is release into the system after six or seven minutes, just as it is with a normally functioning pancreas (8). The pump can be worn in many ways including as a waistband, as a belt or put in the pocket. The patient chooses the mechanisms comfortable for his or her needs. Further, with the advancement of technology, wireless pumps have been developed (18). The pumps provide rapid acting insulin to the body but one is required to program the machine while eating to ensure the provision of the right bolus dose. The bolus dose helps to take care of the food and correct the blood sugar levels if one consumes more than the required (3). The use of pumps is more preferred to injections in the management of blood sugar levels. Other treatment methods include the use of an artificial pancreas, use of high blood pressure medications and cholesterol lowering drugs. The most important thing is to provide a certain level of insulin to the body to take care of the pancreatic malfunction and allow the normal functions of the body to take place as normally as possible (13). 


\section{The Impact of Insulin Pumps in the Management of Type I Diabetes}

History. Several studies have been conducted on the effectiveness of the use of insulin pumps in the management of type I diabetes today. Different topics on the use of this mechanism to administer insulin into the body for patients suffering from type I diabetes are researched (20). However, the immunological impact of this treatment mechanism has not been keenly discussed in literature. The development of the first insulin pump was achieved through ingenuity and teamwork of Yale doctors in the year 1979 (19). The use of the pump allowed the patients to receive continuous levels of insulin during meal times rather than administering one large dose. The use of continuous administration of the insulin mimicked the pancreatic function allowing the body to respond normally to the intervention mechanism (30). The pump was tested on seven children who were kept under close observation at the hospital and the doctors discovered that the use of the new device kept the blood sugar level steady for a long period in comparison to other methods of administration (36). The mechanism has further been developed and it is one of the most effective therapies for people suffering from type I diabetes in the world today.

\section{Advantages}

The insulin pump can be altered to multiply the amount of insulin delivered to the body. The users of this mechanism argue that the se of insulin pumps provides the patient with a better quality of life in comparison to other therapies (12). Further, the pump provides rapid acting insulin thus eliminating the need for exercise that was previously required to maintain blood sugar levels for people. The injections often provide slow acting insulin and this cannot take care of the basal rates necessary to manage after meals situations (25). The use of pumps is more convenient that using injections especially in public. The pumps are designed in a way that gives the patient privacy. Some people many fail to take the required dosage of insulin because they are uncomfortable using the injections in public, but with the insulin pump, this problem is eliminated (21). The pumps are designed in a way that allows proper calculation of the amount of insulin administered to the body thus making it easy for the patient to manage the condition effectively. Further, doctors and healthcare personnel can use this information to accurately determine the insulin needs of the patient and provide the ideal dosage for a better living (23).

Most of the complications affecting people suffering from type I diabetes occur because of the improper administration of insulin. It is easy to make a mistake with the syringe especially for young people. It is important to understand that type I diabetes is more prevalent among young people and hence the need to find a non-manual mechanism of administering the insulin doses for effective treatment (28). The pumps make it possible to administer precise amounts that are impossible to achieve with the use syringes and needles. As a result, the use of pumps provides a better control of hemoglobin and blood sugar levels thus eliminating the occurrence of long-term complications connected to diabetes (34). Additionally, the lack of complications translates to low cost of the disease management for the patient. The recorded history of the insulin pump can be used to determine the trends and the needs of the patient thus improving the quality of life and reducing the disease burden for caregivers. The use of pumps has also been associated with the reduction of neuropathy pain, which is a major complication for people suffering from diabetes (20). Reports have indicated that the use of pumps eliminate resistance to usual treatment among diabetic patients thus making it easy to control the illness.

\section{Limitations}

The use of pumps is ideal but it can be a bit costly for most patients. Further, in case of the pump failure, patients are at a higher risk of suffering from ketoacidosis (28). The pump is a machine like any other and failure can occur at any time. The pump malfunction can be because of battery discharge, heat exposure that inactivates the insulin, the depletion of the reservoir, loose tubing, and insulin leakage among other complications. The use of this mechanism is required to constantly monitor the blood sugar level to ensure that the pump is functioning normally, which can be time consuming and overwhelming.

Pumps are carried around; therefore, the patient can be limited in operating in some ways if he or she does not devise a mechanism to keep the pump in place. For instance, students can find it hard to participate in sports and keep the insulin pumps in place. Scar tissue building around the catheter insertion area is a common problem among the uses of insulin pumps (9). Allergic reactions as the body adjusts to the infusion set can also affect the patient's quality of life. Moreover, care must be taken when refilling and washing the insulin pump to prevent changes in prescription and dosage information.

\section{Immunological Impacts of Using Insulin Pumps}

In their study, Geerlings and Hoepelman (15) argue that patients with diabetes have a lower immunity levels in comparison to those living without the disease. The complications experienced by this group of people are severe 
than in normal people. The authors associated this prevalence of diseases and severity of conditions to defects in the immunity system of patients with type I diabetes (15). The condition causes disturbances in humeral innate immunity thus leading to the prevalence of infections among patients of diabetes. A better management of the disease leads to the improvement of these cellular thus causing the body to respond effectively to disease causing pathogens. Further, since microorganisms responsible for various infections thrive well in a high glucose environment, the administration of insulin through different mechanisms helps to destroy these organisms thus improving the patient's quality of life (15). Failure to administer insulin can lead to several defects of the innate immune system.

Atkinson, Eisenbarth and Michels (5) argue that the prevalence of type I diabetes has risen over the last few decades. Several methods have been developed to provide the insulin do to the patient one of them being the use of pumps. The insulin pump has been regarded as the most effective administration mechanism that allows the patients to reach their target with much ease. The use of pumps advance glycemic control and reduce hypoglycemia (5). With the use of pumps, the patient and the caregivers are able to constantly monitor the blood sugar thus improving the quality of life for the patients. The monitoring of sugar levels in the body prevents hypoglycemic episodes that often occur when the insulin threshold is not achieved for a period of two hours (5). Although the use of insulin pumps has increased in the last few decades, the immunological response of the body to these therapies is still not well understood. The lack of a clear relationship between the genetic complexity of type I diabetes makes it hard for the scientists to deduce the exact immunological response to insulin pumps.

Rewers and Gottlieb (13) provide an immunotherapy analysis for the prevention and treatment of type I diabetes in their paper. They argue that for a mechanism to work effectively in the management of type I diabetes, one has to have a clear understanding of the basic mechanisms underlying the disease (13). Diabetes is an autoimmune disease that is caused by the interaction of environmental and genetic factors. However, it is unclear which environmental triggers cause the development of type I diabetes. After the commencement of islet autoimmunity, patients experience an extended pre-clinical period that allows for secondary prevention. Tertiary prevention mechanisms such as the administration of insulin through pumps use a number of immunomudulatory agents. The agents are effective for patients with established diabetes, but can also be given to patients with dysglycemia and normoglycemic pre-type I diabetes after being proven safe (13). The use of pumps can interfere with the preservation of C-peptide; therefore, the use of insulin pumps should be applied with a lot of care because they can create a point-of-no return in the immune system of the patient once used.

Fineberg et al. (14) argue that regardless of the effectiveness of the therapeutic insulin, several immunological complications do occur among all number of patients. Insulin autoantibody is usually detected in individuals who have a likelihood of developing type I diabetes. Although immune tolerance occurs after a long use of several therapies, the problem can be overcome. The immune complications arising from the use of insulin pumps and other treatment mechanisms depend on the patient's age, genes, and the site of insulin delivery among other aspects (14). The development of insulin antibodies affects glucose control, dose requirements and creates incidences of hypoglycemia. Studies indicate that there is no connection between the developments of insulin antibodies and fatal risks for type I diabetic patients (14). Different insulin administration mechanism are accompanied by several immunological complications, but the use of insulin pumps has not been singularly linked with major complications

The use of insulin pumps affects the glucose homeostasis, hormones, metabolites and the quality of life (27). People who use the insulin pumps have manageable blood glucose levels. Further, the use of insulin pumps leads to a nearly normalized diurnal patterns of FFA and other plasma bodies. People treated through this mechanism live near normal lives with a few fluctuation of glucose level during morning hours. According to a recent study, people treated with the insulin pumps developed ketoacidosis during the therapy while those under a different treatment mechanism were hospitalized with hypoglycemic coma (27). The study concluded that the use of insulin pumps for about six months helps to bring normalcy to the level of glucose in the body characterized by changes in the diurnal patterns of plasma insulin. However, caregivers should take note of the high morning values to achieve better results using the intervention mechanism.

Patients who develop complication after using other treatment mechanisms are brought to a stable position by the use of insulin pumps. For instance, a five-year-old patient developed uncontrollable reaction to insulin and after diagnosis, she was found to have positive intradermal skin tests to regular insulin (24). The patient was put under antihistamine and other insulin administration mechanism without any relief. The use of insulin pumps helped to 
stabilize the patient and restore the glucose balance in the blood. The use of the pumps is therefore ideal for people with allergies towards insulin and for insulin desensitization among children.

Insulin pumps are becoming quite common and are widely used by type I diabetic patients in the world today. The pumps have been praised for their ability to control blood glucose effectively in comparison to other mechanisms of treatment (24). However, regardless of their effectiveness, the insulin pumps are not fit for everyone. The machine guarantees the supply of insulin to the body 24 hours in a day and it is small enough for one to carry. The machine is set to release the right dosage of insulin into the system after a period thus allowing the patient to live a comfortable, near normal life.

The basal insulin is programmed to release enough quantities to supply the needs of the patient at all times. During meal times, the dosage is changed to deal with the inflated levels of glucose at the time. The insulin pump provides rapid-acting type of the hormone throughout the day and it is able to take care of all the needs of the patient (18). Some pumps are fitted with sensors that measure the blood sugar levels thus increasing the effectiveness of the therapy.

Although insulin pumps are convenient, they are not as easy as they sound to all people. One must be willing to manage the illness on a daily basis to qualify as an eligible candidate for the insulin pump intervention mechanism. The use of the pumps requires one to count the amount of carbohydrates in every meal to adjust the dosage of the insulin accordingly (36). Further, one has to check glucose level about four times a day to ensure that the right quantity of insulin is delivered into the system. the willingness to miss meals, check basal rates, and calculate insulin-carbohydrate ratios are some of the things that people using the insulin pump must be willing to do to ensure that the therapy does not produce negative immunological impacts.

\section{References}

1. Akerblom, Hans, Outi Vaarala, Heikki Hyoty, Jorma Ilonen, Mikael Knip. "Environmental factors in the etiology of type 1 diabetes." AJMG 115. 1 (2002): 18-29. Print.

2. Aronson, J. K. Meyler's side effects of endocrine and metabolic drugs. Amsterdam, NL: Elsevier, 2009. Print.

3. Aronson, J. K. Side effects of drugs. Amsterdam: Elsevier, 2005. Print.

4. Atkilt, Helen, Muluken Turago and Balewgizie Tegegne. "Clinical Characteristics of Diabetic Ketoacidosis in Children with Newly Diagnosed Type 1 Diabetes in Addis Ababa, Ethiopia: A Cross-Sectional Study." PLOS One, (2017): 1-9. Print.

5. Atkinson, Mark A, George S Eisenbarth, and Aaron W Michels. "Type 1 Diabetes." Lancet 383.9911 (2014): 69-82. PMC. Print.

6. Atkinson, Mark A. "The Pathogenesis and Natural History of Type 1 Diabetes." Cold Spring Harbor Perspectives in Medicine 2.11 (2012): a007641. PMC. Web. 1 May 2017.

7. Bruttomesso, Daniela, and Giorgio Grassi. Technological advances in the treatment of type 1 diabetes. Basel New York: Karger, 2015. Print.

8. Byng, Karen. Insulin pump use in Australia. Canberra: Australian Institute of Health and Welfare, 2012. Print.

9. Casqueiro, Juliana, Janine Casqueiro, and Cresio Alves. "Infections in Patients with Diabetes Mellitus: A Review of Pathogenesis." Indian Journal of Endocrinology and Metabolism 16.Suppl1 (2012): S27-S36. Print.

10. Chantelau, Ernst, Ruth Prator, and Jorg Prator. "Insulin-induced localized lipoatrophy preceded by shingles (herpes Zoster): a case report." Journal of medical case reports 8. 223 (2014): 1-5. Print.

11. Chiang, Jane, Sue Kirkman, Lori Laffel, and Anne Peters. "Type 1 Diabetes through the Life Span: A Position Statement of the American Diabetes Association." Diabetes care 40.5 (2014): 2034-2054. Print.

12. Crowe, Lynn, and Julie Stachowiak. The diabetes manifesto : take charge of your life. New York: DemosHealth, 2010. Print.

13. Ehrman, Jonathan K., et al. Clinical exercise physiology. Champaign, IL: Human Kinetics, 2013. Print.

14. Fineberg, Edwin, Thomas Kawabata, Deborah Finco-Kent, Robert Fountaine, Gregory Finch, and Alan Krasner. "Immunological responses to exogenous insulin." Endocrine reviews 28.6 (2007): 625-652. Print.

15. Geerlings, Sizanne and Andy Hoepelman. "Immune dysfunction in patients with diabetes mellitus (DM)." Pathogens and Disease 26. 3-4 (1999): 625-265. Print.

16. Gerstein, Hertzel C., and R B. Haynes. Evidence-based diabetes care. Hamilton, Ont: B.C. Decker, 2001. Print.

17. Hassan, Gamal Abdulrhman et al. "Role of Immune System Modulation in Prevention of Type 1 Diabetes Mellitus." Indian Journal of Endocrinology and Metabolism 16.6 (2012): 904-909. Print. 
18. Hussain, Syed S., and Nick Oliver. Insulin pumps and continuous glucose monitoring made easy. Edinburgh New York: Elsevier, 2016. Print.

19. Jameson, J L., et al. Endocrinology: adult \& pediatric. Philadelphia, PA: Elsevier/Saunders, 2016. Print.

20. Jameson, J L., Leslie J. DeGroot, and D. M. Kretser. Endocrinology: adult and pediatric. Philadelphia: Saunders/Elsevier, 2010. Print.

21. Kamata, Yuji, Koji Takano, Eriko Kishihara, Michiko Watanabe, Raishi Ichikawa, Masayoshi Shichiri. "Distinct clinical characteristics and therapeutic modalities for diabetic ketoacidosis in type 1 and type 2 diabetes mellitus." The journal of diabetes and its complications 31.2 (2017): 468-472. Print.

22. Lehuen, Agnes, Julien Diana, Paola Zaccone, and Anne Cooke. "Immune cell crosstalk in type 1 diabetes." Natural reviews immunology 10 (2010): 501-513. Print.

23. Levy, David. Type 1 diabetes. Oxford: Oxford University Press, 2016. Print.

24. Maahs, David M., Lauren A. Horton, and H. Peter Chase. "The Use of Insulin Pumps in Youth with Type 1 Diabetes." Diabetes Technology \& Therapeutics 12.Suppl 1 (2010): S-59-S-65. PMC. Print.

25. Misra, Shivani and Nick Oliver. "Diabetic ketoacidosis in adults." BMJ, 351 (2015): 1-8. Print.

26. Onyiriuka, Alphonsus, and Emeka Ifebi. "Ketoacidosis at diagnosis of type 1 diabetes in children and adolescents: frequency and clinical characteristics." Journal of diabetes \& Metabolic disorders, 12.47 (2013): 1-5. Print.

27. Pickup, John. "Insulin-pump therapy for type I diabetes mellitus." The New England journal of medicine 366 (2012): 1616-24. Print.

28. Platt, Elizabeth, Jerrold S. Olshan, and Maryann Waterman. 100 questions \& answers about your child's type 1 diabetes. Sudbury, MA: Jones \& Bartlett Learning, 2012. Print.

29. Razavi, Zahra. "Frequency of Ketoacidosis in Newly Diagnosed Type 1 Diabetic Children." Oman Medical Journal 25.2 (2010): 114-117. Print.

30. Reece, E A., et al. Diabetes mellitus in women: adolescence through pregnancy and menopause. Philadelphia, Pennsylvania: Lippincott Williams \& Wilkins, 2004. Print.

31. Rewers, Marian, and Peter Gottlieb. "Immunotherapy for the Prevention and Treatment of Type 1 Diabetes: Human Trials and a Look into the Future." Diabetes Care 32.10 (2009): 1769-1782. Print.

32. Rodgers, Jill. Using insulin pumps in diabetes: a guide for nurses and other health professionals. Chichester, England Hoboken, NJ: John Wiley, 2008. Print.

33. Sarikonda, Ghanashyam, Jeremy Pettus, Sowbarnika Sachithanantham, Sonal Phatak... and Mathias Herrath. "Temporal intra-individual variation of immunological biomarkers in type I diabetes patients: implications for future use in cross-sectional assessment. PLOS One 8.11 (2013): 1-13. Print.

34. Sperling, M. Type 1 diabetes: etiology and treatment. Totowa, N.J: Humana Press, 2003. Print.

35. Steineck, Isabelle, Jna Cerderholm, Bjorn Eliasson, Araz Rawshani...and Soffia Gudbjornsdottir. "Insulin pump therapy, multiple daily injections, and cardiovascular mortality in 18168 people with type I diabetes: observational study." BMJ 350 (2015): 1-9. Print.

36. Strober, Warren, Susan R. Gottesman, and Richard Coico. Immunology: clinical case studies and disease pathophysiology. Hoboken, N.J: Wiley-Blackwell, 2009. Print.

37. Uchigata, Yasuko. "Can Restoring Immune Balance Be the Ultimate Therapy for Type 1 Diabetes?" Journal of Diabetes Investigation 7.6 (2016): 819-821. Print.

38. Walsh, John, and Ruth Roberts. Pumping insulin: everything you need to succeed on an insulin pump. San Diego, California: Torrey Pines Press, 2012. Print.

39. William, Isley, and Mark Molitch. "Type 1 Diabetes." J Clin Endocrinol Metab 90.1 (2005): E2. doi: 10.1210/jcem.90.1.9996 